\title{
Mycelium Macromorphology and Growth Characteristics of Culinary-Medicinal Mushroom Flammulina velutipes (W.Curt.: Fr.) Singer
}

\author{
Suzanna M. Badalyan E๐ Carmen Z. Sakeyan \\ Laboratory of Fungal Biology and Biotechnology, Yerevan State University, Department of \\ Botany, 1 Aleg Manoogian St., 375025, Yerevan, Armenia
}

Recent advances in biotechnology increased the role of different groups of organisms for obtaining biologically active compounds (BAC) in the development of health-enhancing biopreparations and dietary supplements. Medicinal Basidiomycetes mushrooms are promising in the production of new biopharmaceuticals and as food additives. However, the screening of macro- and micromorphological characteristics and optimal growth conditions of mycelium of these species, selection of fast growing strains/active producers of BAC are preliminary steps in their biotechnological cultivation protocol.

The object of our study is a well known woodinhabiting, culinary-medicinal mushroom known as
Flammulina velutipes. Based on bibliographic data and our own observations, this species contains different groups of active compounds (polysaccharides, protein/glucan complexes, sterols, lectins, phenolic compounds, etc.) and substrate-specific enzymes. The large spectrum of pharmacological activity (immune-modulating, antitumor, antifungal, antiviral, antibacterial, fibrinolytic, thrombolytic, hypolipidic, antioxidant, spasmolytic, etc.) of $F$. velutipes' metabolites was reported.

The 21 strains of $F$. velutipes, separated from different geographical regions (Armenia: 17 strains, France: 3, and Russia: 1) and from different wood substrates (poplar: 1 strain, oak: 8 strains, grapes rod: 1 strain, ash-tree: 1 strain, pin-tree: 2 strains, 
and leaves-tree stumps: 8 strains) were involved in the screening of growth and macro-morphological characteristics. Two formulas were used to calculate the colony growth rate (GR) and growth coefficient (GC): $G R=\Delta d / \Delta t$ and $G C=d g h / t$, where $d=\mathrm{a} d i-$ ameter of colony growth, $t=$ time, $g$ and $h$ represent the density and height of colony, respectively. The mycelial growth characteristic and morphology were analyzed after incubation of cultures on $2 \%$ maltextract agar medium (MEA, $\mathrm{pH}=5.5$ ) at $25^{\circ} \mathrm{C}$. The measurements were taken for 11 days. The observations continued 2 months until primordia or fruiting body formation.

Based on GR indicators, all screened $F$. velutipes strains were divided into three main groups: I: GR $<12 \mathrm{~mm} /$ day (9.5\%), II: $12-14.5 \mathrm{~mm} /$ day (47.6\%), and III: GR > $14.5 \mathrm{~mm} /$ day (42.9\%). The first group involved one Russian (R-9) and one Armenian (II4) strains. The French strains possessed average GR and belonged to the second group.

Two morphological types of colony $\mathrm{A}$ and $\mathrm{B}$ within $F$. velutipes strains were described. Type A colony is farinaceous and white and later turns yellowish. The agar has yellow-brown pigmentation. Fruiting body formation was mentioned for all strains during 1-1.5 months of observation. Type A is typical for F. velutipes and includes average(10 strains) and fast-growing (4 strains) separated from fruiting bodies from Armenia and France. They formed two colony "subtypes" with dense (A1) and sparse (A2) mycelium, respectively. Type
$\mathrm{B}$ is a slowly growing morphotype of the F. velutipes colony with wadded and dense mycelium. The pigmentation of agar is nearly absent. The fruiting of this mycelial type was not mentioned during our observation. Type B was observed in one Russian (R-9, ash-tree) strain. However, a mixed A-B type of colony was also observed in three French and three Armenian (II-2, II-3, II-4) strains, separated from fruiting bodies collected on ash-tree and oak, respectively.

All screened strains on the $6^{\text {th }}$ day of mycelial growth were divided into three groups based on GC indicators: I: GC <30 (28.5\%), II: GC $=30-60$ (38.1\%), and III: GC >60 (23.8\%). The first group involved Armenian strains with type A2, whereas group II was composed of the strains with A1 morphotype. French, Armenian (II-4), and Russian (R9) strains were involved in group III.

We concluded that two A and B species-specific morphological types or ecotypes of colonies described in our collection of $F$. velutipes correlated with their substrate nature and geographical origination.

\section{AKNOWLEDGMENTS}

This work is financially supported by the grants of the Ministry of Science and Education of Armenia (\# 0104), NATO (\# FEL.RIG. 980764), DAAD (Pr. No 548.104401.174), and ANSEF (\# 04-NSbiotech-814-73). 